MS18-P09

\section{High pressure pair distribution function study of amorphous silica in helium}

Jerome Rouquette $^{1}$, Julien Haines ${ }^{1}$, Daniel Bowron ${ }^{2}$, David Keen ${ }^{2}$, Mathieu Kint ${ }^{3}$, Coralie Weigel ${ }^{3}$

1. ICGM, UMR CNRS 5253, Université de Montpellier. CC01504, 34095 Montpellier, Montpellier, Fraance, France

2. ISIS, Rutherford Appleton Laboratory, Harwell Oxford, Didcot, OX11 0QX, UK, Didcot, OX11 0QX, United Kingdom

3. L2C, UMR CNRS 5221, Université de Montpellier. CC069, 34095 Montpellier, Montpellier, France

email: jerome.rouquette@umontpellier.fr

Glasses are used for a large number of technological and everyday applications. Among them, the system that has been most widely investigated is amorphous silica $\left(\mathrm{a}-\mathrm{SiO}_{2}\right)$. The structure of $\mathrm{aSiO}_{2}$ consists of corner-sharing $\mathrm{SiO}_{4}$ tetrahedra which form $n$-fold rings (with $n \geq 2$, in a-SiO $\mathrm{Si}_{2}$ mostly $n=4,5,6,7$, 8). The absence of Bragg reflections in diffraction experiments precludes the use of traditional crystallographic techniques to determine their atomic structure. However, it is clear that these materials possess well-defined local structure on the nanometer scale, which is often linked with their physical properties. Local structure can be obtained using x-ray total scattering from which the distribution of interatomic separations can be measured via the pair distribution function (PDF). High-quality PDFs require high-energy photons and access to large scattering angles to measure data to high values of momentum transfer $(Q=4 \pi \sin$ $\theta / \lambda)$.

It was recently discovered that when helium or argon are used as pressure transmitting medium, a-SiO ${ }_{2}$ exhibits a completely distinct compression behavior[1]. The compressibility of a- $\mathrm{SiO}_{2}$ drastically decreases in a helium medium which can easily be understood by the penetration of helium atoms $\left(r_{\mathrm{He}}=1.3 \AA\right.$ ) in $n$-fold rings (with $n \geq 6, \mathrm{r}_{6 \text {-fold }}$ ring $=1.5 \AA$ ) which prevents collapse of the rings, contrary to $\operatorname{argon}\left(r_{\mathrm{Ar}}=1.88 \AA\right)$, and therefore the densification of the material; based on a poromechanics model [2], up to 0.53 mole $\mathrm{He}$ can be incorporated under pressure. In order to determine the structural change dependence of helium adsorption on $\mathrm{a}_{-} \mathrm{SiO}_{2}$, high pressure PDF measurements were undertaken.

High-pressure high-quality data were obtained using $61 \mathrm{Kev}$ photons up to $22 \AA^{-1}$. The total scattering data was refined by the reverse Monte Carlo (RMC) method [3] in order to obtain changes to bond-angle distributions and network topology of $\mathrm{a}-\mathrm{SiO}_{2}$ in helium as a function of pressure. Partial PDFs were obtained and permitted to understand the structural modifications induced by the helium adsorption at high pressure which consist in: i) a broadening of the $\mathrm{Si}-\mathrm{Si}$ bond distribution(inter-tetrahedra distances) in the first and second coordination shells; as a consequence appearance of a new contribution is also observed above $3 \AA$ for the Si-O PDF, ii) a decrease in the O-He distance distribution with increasing pressure which becomes more ordered as a result of helium confinement.
References:

[1 ] T. Sato, N. Funamori, and T. Yagi, Nat. Commun. 2 (2011).

[2] M. G. Tucker, D. A. Keen, M. T. Dove, and K. Trachenko, Journal of Physics-Condensed Matter 17, S67 (2005).

[3] B. Coasne, C. Weigel, A. Polian, M. Kint, J. Rouquette, J. Haines, M. Foret, R. Vacher, and B. Ruffle, J Phys Chem B 118, 14519 (2014).

Keywords: PDF study, amorphous silica, helium asorption 